\title{
Spectrum of Hematological Diseases in West Bengal, India: Experience from a Tertiary Care Center
}

\author{
Ankit Jitani, Prakas Kumar Mandal*, Prantar Chakrabarti, Shuvra Neel Baul, Rajib De, Tuphan Kanti Dolai
}

Ankit Jitani, Prakas Kumar Mandal*, Prantar Chakrabarti, Shuvra Neel Baul, Rajib De, Tuphan Kanti Dolai

Department of Hematology, Nil Ratan Sircar Medical College, Kolkata-700014, INDIA.

\section{Correspondence}

Dr. Prakas Kumar Mandal

Assistant Professor, Department of Hematology, Nil Ratan Sircar Medical College, Kolkata-700050, INDIA.

Phone no: +91-9433345001

Email: prakas70@gmail.com

\section{History}

- Submission Date: 11-09-2018;

- Revised Date: 22-10-2018,

- Accepted Date: 22-01-2019;

DOI : 10.5530/ijmedph.2019.1.6

Article Available online

http://www.ijmedph.org/v9/i1

\section{Copyright}

(C) 2019 Phcog.Net. This is an openaccess article distributed under the terms of the Creative Commons Attribution 4.0 International license.

\begin{abstract}
Background: The epidemiology of hematological diseases in India is largely unknown due to paucity of data. Our centre caters to a major chunk of hematological patients in Eastern India and is one of the largest centers in terms of patient attendance. Objective: The objective of this study was to analyze the OPD data to decipher the hematological disease spectrum in Eastern India. Methods: Hospital data of hematology OPD from July 2015 to June, 2018 was retrived from the hospital information management system. The number of new and follow-up (old) patients attending the OPD were analyzed to know the disease pattern in terms of various benign and malignant hematological disease was assessed. Results: The total number of OPD consultations for the three years was 78809, of which $21851(27.7 \%)$ were new cases and $56958(72.3 \%)$ were follow-up (old) visits. Disease characterization could be done for 15517 (71.01\%) patients; out of these, 73.3\% (11377/15517) were benign and $26.7 \%(4140 / 15517)$ were malignant hematological diseases. Common reasons for consultation in hematology OPD were anemia 9170 cases (59.1\%), leukemia 2544 (16.4\%), bleeding disorder 1908 (12.3\%), lymphoma $946(6.1 \%)$ and myeloma 450(2.9\%). Among the benign diseases, most common diagnosis was nutritional deficiency anemia 4403 (38.7\%) followed by hemoglobinopathy 3868 (34\%), immune thrombocytopenia 944 (8.3\%), aplastic anemia 694 (6.1\%), hemophilia $637(5.6 \%)$, eosinophilia $148(1.3 \%)$ and thrombosis $80(0.7 \%)$. Among the malignant cases, common causes were leukemia 2351 (56.8\%), lymphoma $940(22.7 \%)$ and myeloma 451 (10.9\%). Conclusion: Anemia remains the most common reason for OPD consultation even in a tertiary care hematology centre. The major burden of malignant hematological disease is leukemia.

Key words: Tertiary Care Center, Hematology Disease, Spectrum.
\end{abstract}

\section{INTRODUCTION}

India is a country with a population of 1.21 billion as par the $15^{\text {th }}$ National Census Survey in $2011 .{ }^{1}$ Considering the population load and diversity, prevalence of hematological diseases in India should be alarming. However, the distribution of these diseases in our country is largely unknown. The reason for this may be multifactorial. Firstly, there are not many dedicated set-ups providing hematology services to the population. Secondly, the field of hematology has evolved tremendously over recent years; advanced laboratory and molecular diagnostic techniques has become imperative in making precise diagnosis, the facility for which are either not available or not accessible to our population especially those coming from distant rural areas. Thirdly, the health services are offered by multiple stakeholders. Public sector is overwhelmed with huge patients load and not many centers are able to maintain adequate data and private sector, which comes at a cost and are available to only a particular segment of the society. ${ }^{1}$

The center at which this study was conducted is a state government set-up providing dedicated hematology services to the people of West Bengal as well as the neighboring states in Eastern India. It is one of the largest centers in terms of patient attendance in this region. Due to the availability of a good laboratory diagnostic facility, most of the patients are offered a concrete diagnosis with precise disease characterization, which makes the analyses of the disease spectrum possible. There is dearth of data pertaining to the distribution of the hematological disease in this region and the morbidity as well as mortality associated with the malignant as well as the benign hematological disease, especially disease with a genetic basis, is immense. Hence, the hospital data of a tertiary level hematology facility was analyzed to gauge into the spectrum of patients visiting health care facility for consultation pertaining to hematological issues. The aim was to analyze the pattern and distribution of hematological disease in Eastern India in general and the state of West Bengal in particular.

\section{MATERIAL AND METHODS}

This was a descriptive study undertaken in the department of Hematology, Nilratan Sircar Medical College and Hospital. This hospital is located in the 
central part of the metropolitan city of Kolkata next to a major railway station and caters to the healthcare needs of not only the state of West Bengal but the neighbouring state and country. OPD services are provided for five days in a week, which includes a dedicated hemoglobinopathy clinic, hemato-oncology clinic and bleeding disorder clinic and on a given day, he number of patient visiting the OPD varies from 150 to 250 cases. The study was undertaken for a period of three years from July 2015 to June, 2018. Out-patient data from the hospital information system was retrived and the number of new and follow-up patient attending our OPD for the period was retrospectively analyzed. All patients visiting the OPD for consultation during the study period for any reason was included. A patient visiting the OPD on multiple occasion was considered a single case during data analysis. For representation of distribution of disease, the cases for which concrete diagnosis could be confirmed by necessary investigations were included and cases that could not be characterized in any disease entity, either due to failure of the patient to follow-up or due to refusal to undergo a diagnostic procedure were excluded from the study. The institutional ethical committee clearance was obtained prior to data collection.

The patients were divided into new patients coming for first consultation and follow-up patients and expressed in terms of percentage. The number of new patients for whom disease characterization could be done was calculated. Among all these patients who were characterized, the disease distribution of benign and malignant condition was done in terms of percentage. Among the benign diseases, distribution of anemia of different types, hemoglobinopathy, bleeding disorder including platelet and coagulation disorder and other rare condition like thrombosis and eosinophilia were analyzed. Among the malignant conditions, we analyzed the data in terms of leukemia, lymphoma, myeloma and other rare conditions. Results were expressed in terms of absolute number or percentage.

\section{RESULTS}

The data represented all age group of patients, including pediatric, adolescents, adult and geriatric age group. The total number of OPD visits in 3 years was 78809, out of which 21851 (27.7\%) were new cases and 56958 (72.3\%) were follow-ups. Among the new cases, disease characterization could be done for $71.01 \%$ (15517/21851) patients. Out of these cases, $73.3 \%$ (11377/15517) were benign and 26.7\% (4140/15517) were malignant hematological diseases. Common reasons for consultation in hematology OPD, as shown in Table 1, were anemia 9170 (59.1\%), leukemia 2351 (16.4\%), bleeding disorder 1908 (12.3\%), lymphoma 940 (6.1\%) and myeloma 451 (2.9\%).

Among the benign diseases, the most common diagnosis was nutritional anemia $4403(38.7 \%)$ of which iron deficiency were 3698 (32.5\%) and rest 705 (6.2\%) were combined nutritional deficiency anemia hemoglobinopathy 3868 (34\%) was the next common cause of anemia. Among them, $2412(62.38 \%)$ were homozygous or compound heterozygous and $1456(37.62 \%)$ were heterozygous. The distribution of hemoglobinopathy is shown in Table 2. The most common major phenotype was HbE $\beta$ thalassemia which comprised 1318 (34.08\%) of all hemoglobinopathies followed by $\beta$-thalassemia major at $722(18.67 \%)$. HbEE homozygous were the next common with 305 (7.88\%) and rest 68 (1.76\%) were others consisting of HbSS and other compound heterozygous. Among the traits, 723 (18.69\%) were $\beta$-thalassemia trait and 604 (15.62\%) were $\mathrm{HbE}$ trait. $\mathrm{HbS}$ trait and $\mathrm{HbD}$ trait comprised 87 (2.24\%) and $11(0.27 \%)$ respectively and rest $19(0.49 \%)$ were others including $\delta \beta$-thalassemia trait and HPFH heterozygous. Other hemolytic anemia comprised 318 $(2.8 \%)$ cases, among which the most common diagnosis was autoimmune hemolytic anemia $171(1.5 \%)$ and rest of the $147(1.3 \%)$ cases included membranopathies, enzymopathies and other rare causes of hemolytic anemia.

\begin{tabular}{|c|c|c|}
\hline Disease type & $\begin{array}{l}\text { Number of } \\
\text { cases } \\
(n=15517)\end{array}$ & $\begin{array}{l}\text { Percentage } \\
\text { distribution }\end{array}$ \\
\hline Anemia & 9169 & $59.09 \%$ \\
\hline Leukemia & 2548 & $16.42 \%$ \\
\hline Bleeding disorder & 1889 & $12.17 \%$ \\
\hline Lymphoma & 941 & $6.06 \%$ \\
\hline Myeloma & 450 & $2.9 \%$ \\
\hline Eosinophilia & 109 & $0.7 \%$ \\
\hline $\begin{array}{l}\text { Splenomegaly (unclassifiable } \\
\text { hematologically) }\end{array}$ & 97 & $0.62 \%$ \\
\hline Thrombotic disorder & 79 & $0.5 \%$ \\
\hline Others & 225 & $1.45 \%$ \\
\hline
\end{tabular}

\begin{tabular}{|c|c|c|}
\hline Type of hemoglobinopathy & $\begin{array}{c}\text { Overall } \\
\text { distribution }\end{array}$ & $\begin{array}{l}\text { Sub-category } \\
\text { distribution }\end{array}$ \\
\hline $\begin{array}{c}\text { Homozygous and compound } \\
\text { heterozygous }\end{array}$ & \multicolumn{2}{|c|}{$62.38 \%$} \\
\hline $\mathrm{HbE} \beta$ & $34.08 \%$ & $54.64 \%$ \\
\hline$\beta$-thalassemia major & $18.67 \%$ & $29.92 \%$ \\
\hline HbE disease & $7.88 \%$ & $12.62 \%$ \\
\hline Others & $1.76 \%$ & $2.81 \%$ \\
\hline Heterozygous & \multicolumn{2}{|c|}{$37.62 \%$} \\
\hline$\beta$-thalassemia trait & $18.69 \%$ & $49.69 \%$ \\
\hline $\mathrm{HbE}$ trait & $15.62 \%$ & $41.52 \%$ \\
\hline $\mathrm{HbS}$ trait & $2.24 \%$ & $5.97 \%$ \\
\hline $\mathrm{HbD}$ trait & $0.27 \%$ & $1.50 \%$ \\
\hline Others & $0.49 \%$ & $1.30 \%$ \\
\hline
\end{tabular}

Aplastic anemia was the next common cause of anemia with 693 (6.1\%) cases and it was the most common cause of pancytopenia requiring OPD consultation. Among the bleeding disorder, the most common entity was immune thrombocytopenia 944 (8.3\%), followed by hemophilia 637 (5.6\%) and other rare bleeding disorders 227 (2\%). Among hemophilia, $87.3 \%$ were hemophilia A and $12.7 \%$ were hemophilia $\mathrm{B}$. The other rare entities comprised cases of eosinophilia 148 (1.3\%) and deep vein thrombosis in $80(0.7 \%)$.

Among the malignant diseases, leukemia far exceeded the other malignancies comprising 2351 (56.81\%) of the total 4140 cases. Acute leukemia comprised 1312 (31.7\%) of all hematological malignancies and 55.78\% among all leukemia. Among the acute leukemias, ALL was more common with 780 (59.5\%) compared to AML (excluding APML) which comprised 457 (34.83\%), while APML comprised 58 (4.4\%) of all leukemia. Other rare variant like mixed phenotypic leukemia were 17 (1.2\%). CML and CLL comprised $902(21.8 \%)$ and 137 (3.3\%) of all malignant cases and $38.48 \%$ and $5.74 \%$ of the leukemia cases respectively (Table 3 ). MPN were $194(4.7 \%)$ of all hematological malignancies. Lymphoma was the $2^{\text {nd }}$ most common malignant disorder with 940 (22.7\%) cases among which non-Hodgkins lymphoma cases were 490 (52.2\%) and Hodgkins 


\begin{tabular}{|c|c|c|}
\hline Leukemia types & $\begin{array}{l}\text { Overall distribution } \\
\text { among all malignant } \\
\text { cases }\end{array}$ & $\begin{array}{l}\text { Distribution of cases } \\
\text { among all leukemias }\end{array}$ \\
\hline Acute Leukemia & $31.67 \%$ & $55.78 \%$ \\
\hline $\begin{array}{l}\text { AML (excluding } \\
\text { APML) }\end{array}$ & $11.03 \%$ & $19.45 \%$ \\
\hline APML & $1.40 \%$ & $2.47 \%$ \\
\hline ALL & $18.86 \%$ & $33.19 \%$ \\
\hline Others & $0.38 \%$ & $0.67 \%$ \\
\hline Chronic leukemia & $25.1 \%$ & $44.22 \%$ \\
\hline CML & $21.8 \%$ & $38.48 \%$ \\
\hline CLL & $3.3 \%$ & $5.74 \%$ \\
\hline
\end{tabular}

lymphoma were 450 (47.8\%). Rest of the malignant cases were multiple myeloma 451 (10.9\%), MDS and MDS/MPN 161 (3.9\%).

\section{DISCUSSION}

The HMIS data of the Hematology department for three years from June 2015 to July 2018 was collected and analyzed with the aim to understand the distribution of hematological diseases in the population visiting the OPD of haematology department of a tertiary hospital. Disease characterization could be done for $71 \%$ of the patient visiting for consultation. Rest of the $29 \%$ cases included patients for whom a precise hematological diagnosis could not be made that included patients who either did not follow-up after the first/second visit and hence diagnosis could not be confirmed, or patients who refused a diagnostic procedure/investigation, or cases that were referred to the hematology OPD from other speciality and superspeciality departments for procedural fitness or for complete blood count and or coagulation profiles or advice for other hematological tests which are done in our departmental laboratory.

The most common reason for consultation in the hematology OPD was anemia at $59.1 \%$ with nutritional anemia being the most common cause. According to WHO, there are two billion people with anemia in the world, with half of the anemia due to iron deficiency. ${ }^{2}$ In South East Asia, $66 \%$ children, $60 \%$ women and $36 \%$ men are anemic. ${ }^{3}$ In India, the NHFS-4 data showed that the prevalence of anemia among children of 6 to 59 months age was $58.5 \%$ and among adult (15 to 49 years) male and female were $22.7 \%$ and $53 \%$ respectively, while in West Bengal, the prevalence among children, adult male and female were $54.2 \%, 30.3 \%$ and $62.4 \%$ respectively. ${ }^{4}$ In another study from North India, the prevalence of anemia was $69.6 \%$ with $31 \%$ cases being iron deficiency anemia. ${ }^{5}$ In the present study, $32.5 \%$ of the patients were iron deficiency anemia while $6.2 \%$ were due to combined nutritional deficiency. Malnutrition is a major cause of nutritional anemia in our country. A study done in the school going children in West Bengal showed that $40 \%$ of the adolescent were malnourished and $40 \%$ showed the presence of clinical pallor. ${ }^{6}$ Strategies like fortified food and nutrition supplements may help to control this public health problem. This data however do not represents the community and hence it is not reflective of the disease prevalence, but does emphasize that anemia is the most common reason for OPD consultation even in a tertiary care center and community level screen and preventive strategy needs to be strengthen to reduce the disease burden In a large population based survey of hemoglobinopathy in West Bengal, it was shown that hemoglobinopathy is present in $12.17 \%$ cases and $\beta$-thalassemia trait was the commonest found in $4.60 \%$ patients followed by $\mathrm{HbE}$ trait $(3.02 \%), \beta$ thalassemia major/intermedia (1.66\%) and $\mathrm{E} \beta$ thalassemia (1.16 \%) cases. $^{7}$ A community based study of 50,487 cases showed that the distribution of heterozygous thalassemia was $10.26 \%$, with $\beta$-trait and $\mathrm{HbE}$ trait being the common entities with $6.61 \%$ and
2.78\% cases; and homozygous and compound heterozygous cases were $1.36 \%$, with $\beta$-thalassemia major and $\mathrm{HbE} \beta$ with $0.73 \%$ and $0.42 \%$ cases being the most common. ${ }^{8}$ Another study from Eastern India, tried to look into the prevalence of hemoglobinopathies in the rural population of West Bengal and found that $\beta$-thalassemia trait was the most prevalent and was found in $10.38 \%$ cases, followed by $\mathrm{HbE}$ trait in $4.30 \%$, sickle cell trait in $1.12 \%$ and $\mathrm{Hb} \mathrm{D}$ trait $0.37 \% .{ }^{9}$ Hemoglobinopathy was the second most common reason of OPD consultation with $34 \%$ cases. There are multiple reasons for such high patient load of hemoglobinopathy patients visiting the OPD, mostly comprising of transfusion dependent thalassemia. Firstly, our center is a Nodal Center for the State Thalassemia Control Program and run a dedicated OPD once a week for thalassemia, where patient turn-out ranges from 220-250 per OPD. Second, it being a referral center for thalassemia management, the major patient load comprises of transfusion dependent thalassemia including $\mathrm{E} \beta$ thalassemia and $\beta$-thalassemia major. Third, the distribution of homozygous state far exceeds heterozygous conditions as this is a hospital based data where mostly symptomatic patient comes for consultation and the heterozygous thalassemia are mainly cases that are referred from other specialty/super speciality departments and from different districts and sub-divisional hospitals for evaluation of anemia. This pattern is unlike the community bases study data where the asymptomatic heterozygous thalassemia far exceeds the homozygous patients. ${ }^{8-9}$ The distribution of nutritional anemia and thalassemia in our data is almost equal. The reason for this nearly similar distribution may be that the nutritional deficiency anemia are treated by other government and private hospitals in the region and also by other departments of our institute, but there are only a few centers managing hemoglobinopathy in this part of the country. Most of the suspected hemoglobinopathy attend OPD for diagnosis, which increases the percentage of this condition explaining the nearly similar distribution of thalassemia and nutritional anemia. The data aptly reflects thalassemia as a major disease burden in this part of India, emphasizing on the need to strengthen the screening program as well as prenatal diagnosis, to reduce the burden of transfusion dependent thalassemia.

With regard to inherent bleeding disorder, Manisha $\mathrm{M}$ et al..$^{10}$ evaluated the spectrum in Western India. In their cohort, $95.3 \%$ of the patients had coagulation disorder, of which Hemophilia A comprised $70.5 \%$ of the cases, followed by Hemophilia B (14\%) and vWD (10.8\%). Rest of the bleeding disorders was inherent platelet disorder. In our cohort, the most common cause of bleeding disorder was immune thrombocytopenia with $52 \%$ cases, followed by hemophilia with $32 \%$ cases, of which Hemophilia A comprised 87.3\% cases and Hemophilia B included 12.7\% cases. Rest of the bleeding disorder were other rare bleeding and coagulation defects like vWD, functional platelet defects and other rare coagulation factor deficiency and acquired defects of coagulation. We provide on-demand therapy to hemophilia patients and prophylaxis is limited to children, mainly due to limited resources. This prophylaxis program is supported by the World federation of hemophilia and hemophilia federation of India. The present data could have profound implication in terms of increasing the scope of prophylaxis to adults as well, increasing awareness among health care providers in terms of hemophilia management and increasing the number of centers providing hemophilia care, which will reduce the morbidity in the patients in long run. Shirley MH et al. ${ }^{11}$ in a UK based study looked into the distribution of hematological malignancies in various ethnic groups including Indian. In their data leukemia comprised $31.4 \%$ and lymphoma $68.6 \%$ in patients overall and among the Indian populations, leukemia comprised $24.8 \%$ cases and lymphoma $75.2 \%$ cases. In a hospital based data from the neighboring country of Bangladesh, the distribution of AML was $28.3 \%$, ALL was $14.1 \%$, lymphoma was $20.8 \%$, CML was $18.2 \%$, multiple myeloma was $10.5 \%$ and CLL was $3.7 \% .{ }^{12}$ The present data shows a similar distribution of myeloma and lymphoma but a higher load of acute leukemia. This is probably because of increased number of cases referred from the neighboring areas for diagnosis and manage- 
ment due to the availability of the facilities at our center. Also, there are a number of oncology centers which manage lymphoma and myelomas, but the acute leukemias are mostly referred to institutes specializing in the management of these disease and there are a handful of such institutes in this part of India, thus increasing the load of acute leukemia. Another interesting difference was the distribution of acute leukemia which was markedly different, with ALL being predominant in the present data, unlike AML in theirs. APML is regarded as one of the most curable form of acute leukemia. In the present study, APML comprised $11.26 \%$ of all AML cases. This is similar to the reported incidence of 10-15\% of APML among all the AML cases. ${ }^{13}$ The only way to look into the prevalence of these hematological malignancies is hospital based data from the specialized centers managing these disease. There is not much data available from eastern India which reflects the incidence and prevalence of the malignant hematological disease. The present data shows that the incidence of hematological malignancy in this region in staggering. Strengthening the resourse, both infrastructure and human and increased numbers of specialized centers catering to these patients are required to manage them and provide quality services to the population. The importance of knowing the distribution serves multiple purposes. Firstly, by knowing the pattern, proper health care planning and budget allocation for better patient management is possible. Due to evolution in diagnostic tool and management with targeted therapies, hematology services have become expensive and not widely accessible and available. By knowing the disease pattern, planning in terms of diagnostic and treatment facilities required for managing the patient may be done. Secondly, nutritional anemia is the most prevalent cause of anemia in our data, targeted community screening for these deficiency may help in reduction of the disease burden, reducing the morbidity in the population, especially in the pediatric, geriatric, pregnant and nursing mothers. Thirdly, for genetic conditions like thalassemia and hemophilia community screening to detect carriers may be undertaken. Sate thalassemia control program under the aegis of government of West Bengal is trying to do the same in the field of thalassemia.

The limitation of the study is that this is a hospital based data and it cannot be used to extrapolate the incidence of the disease in the community. Benign diseases may be screened at the community level and rightly so, being done for diseases like nutritional deficiency and hemoglobinopathy. But community level screen is not possible for malignant disease and hospital based data remains the only way to look into the disease incidence and prevalence, explaining the importance of hospital based data in planning the infrastructure required for the management of the patients

\section{CONCLUSION}

Hematological disease is highly prevalent in the community and data regarding distribution of the same is sparse in our country. The number of centers providing hematological services is also limited. Advanced diagnostic tool required for correct diagnosis and management makes the precise diagnosis difficult in centers that lack adequate facility. Benign hematological disease comprise a major disease burden and community level awareness is required to decrease this load. Hospital based data is probably the only way to gauge into the prevalence of malignant hematological disease and the present data show that high prevalence of the same in this region. These diseases are associated with high morbidity and mortality and to control them early diagnosis and management is imperative for which more number of centers providing quality care as well as increase in the health care expenditure to manage these diseases are required. We hope this will turn into reality in future with proper planning and public-private partnership.

\section{ACKNOWLEDGEMENT}

We acknowledge all the laboratory and nursing staff of the Hematology department.

\section{CONFLICT OF INTEREST}

The authors declare no conflict of interesting.

\section{ABBREVIATIONS}

OPD: Out-patient department; HMIS: Hospital management information system; ALL: Acute lymphoblastic; AML: Acute myeloid leukemia; APML: Acute promyelocytic leukemia; CML: Chronic myeloid leukemia; CLL: Chronic lymphocytic leukemia; MPN: Myeloproliferative neoplasms; MDS: Myelodysplastic syndrome; MDS/MPN: Myelodysplastic syndrome/Myeloproliferative neoplasm; NFHS-4: National Family Health Survey 4; vWD: von Willebrand disease.

\section{SUMMARY}

This study presents the spectrum of hematological disease in a atertiary care center. Anemia remains the most common reason for consultation in the OPD, most of which are secondary to nutritional deficiency and thalassemia. The proportion of malignant hematological disease is staggering and number of centers required to treat the patients are lacking. This data reflects urgent need of expansion of infrastructure to deal with these diseases.

\section{REFERENCES}

1. Balarajan Y, Selvaraj S, Subramanian SV. Health care and equity in India. Lancet 2011;377(9764):505-15.

2. WHO, UNICEF, UNU. Iron Deficiency Anaemia: Assessment, Prevention and Control, A Guide for Programme Managers, WHO, UNICEF, UNU, Geneva Switzerland, 2001. Available from http://www.who.int/nutrition/publications/micronutrients/anaemia_iron_deficiency/NHO_NHD_01.3/en/index.html

3. Stoltzfus RJ, Mullany L, Black RE. Iron deficiency anaemia. Comparative Quantification of Health Risks: Global and Regional Burden of Disease Attributable to Selected Major Risk Factors. Geneva: World Health Organization. 2005;1:163-209.

4. National family health survey (NFHS-4) 2012-14. Mumbai: International Institute for Population Sciences (IIPS) and Macro International. 2009. Available from: http://www.rchiips.org/nfhs/nfhs4.shtml.

5. Kumar T, Taneja S, Yajnik CS, Bhandari N, Strand TA. Study Group. Prevalence and predictors of anemia in a population of North Indian children. Nutrition. 2014;30(5):531-7.

6. Dey I, Biswas R, Ray K, Bhattacherjee S, Chakraborty M, Pal PP. Nutritional status of school going adolescents in a rural block of Darjeeling, West Bengal, India. The Health. 2011;2(3):75-7.

7. Mondal SK, Mandal S. Prevalence of thalassemia and hemoglobinopathy in eastern India. A 10-year high-performance liquid chromatography study of 119,336 cases. Asian J Transfus Sci. 2016;10(1):105-10.

8. Mandal PK, Maji SK, Dolai TK. Present scenario of hemoglobinopathies in West Bengal, India: An analysis of a large population. International Journal of Medicine and Public Health. 2014;4(4):496-9.

9. Dolai TK, Dutta S, Bhattacharyya M, Ghosh MK. Prevalence of hemoglobinopathies in rural Bengal, India. Hemoglobin. 2012;36(1):57-63.

10. Manisha M, Ghosh K, Shetty S, Nair S, Khare A, Kulkarni B, et al. Spectrum of inherited bleeding disorders from Western India. Haematologia (Budap). 2002;32(1):39-47.

11. Shirley $\mathrm{MH}$, Sayeed $\mathrm{S}$, Barnes I, Finlayson A, Ali R. Incidence of haematological malignancies by ethnic group in England, 2001-7. $\mathrm{Br} \mathrm{J}$ Haematol. 2013;163(4):465-77.

12. Hossain MS, lqbal MS, Khan MA, Rabbani MG, Khatun H, Munira S, et al. Diagnosed hematological malignancies in Bangladesh- $A$ retrospective analysis of over 5000 cases from 10 specialized hospitals. BMC Cancer. 2014;14(1):438. [doi: 10.1186/1471-2407-14-438]

13. Chen Y, Kantarjian H, Wang H, Cortes J, Ravandi F. Acute promyelocytic leukemia: a population-based study on incidence and survival in the United States, 19752008. Cancer. 2012;118(23):5811-8. 